\title{
Review
}

\section{Import of proteins into mitochondria: a multi-step process}

\author{
Nikolaus PFANNER, Franz-Ulrich HARTL and Walter NEUPERT \\ Institut für Physiologische Chemie, Unjversitāt München \\ (Received March 26, 1988) - EJB 880350
}

Translocation of precursor proteins from the cytosol into mitochondria is a multi-step process. The generation of translocation intermediates, i.e. the reversible accumulation of precursors at distinct stages of their import pathway into mitochondria ('translocation arrest'), has allowed the experimental characterization of distinct functional steps of protein import. These steps include: ATP-dependent unfolding of precursors; specific recognition of precursors by distinct receptors on the mitochondrial surface; interaction of precursors with a general insertion protein ('GIP') in the outer mitochondrial membrane; membrane-potential-dependent translocation into the inner membrane at contact sites between both membranes; proteolytic processing of precursors; and intramitochondrial sorting of precursors via the matrix space ("conservative sorting"). The functional characteristics unveiled by studying mitochondrial protein import appear to be of general interest for investigations on intracellular protein sorting.

Over $90 \%$ of mitochondrial proteins are nuclear-coded and are synthesized as precursor proteins on cytosolic polysomes (for recent reviews, see [1 - 3]). The precursors are then translocated to their functional destination in one of the four mitochondrial compartments (outer membrane, intermembrane space, inner membrane, and matrix). More than ten years ago, it was demonstrated that mitochondrial protein import could occur post-translationally in vivo and in vitro, thereby proving the mechanistic independence of translation and translocation, and that protein import required energy [4-7]. Since then, several important characteristics of mitochondrial protein uptake have been unravelled which we would like to mention in a short historical overview. These include the demonstration of amino-terminal peptide extensions ('presequences') on the precursor proteins $[8,9]$ which are proteolytically processed in the mitochondrial matrix [10]. On the other hand, several precursors have been shown to by synthesized without cleavable peptide extensions [11, 12]. Protein translocation into the inner membrane requires the membrane potential $(\Delta \Psi)$ across the inner mitochondrial membrane $[13-15]$. Several precursors are proteolytically processed in two steps, the second processing activity residing in the intermembrane space [16-18]. The primary sequences of precursor proteins have been determined, demonstrating that the presequences are positively charged $[19,20]$. Precursors imported in vitro have been shown to be assembled into multi-subunit protein complexes [21, 22]. Amino-terminal precursor sequences, either the presequences or amino-terminal portions in the mature part of non-cleavable precursors, have

Correspondence to $\mathrm{W}$. Neupert, Institut für Physiologische Chemie, Universität München, Goethestraße 33, D-8000 München 2, Federal Republic of Germany

Abbreviations. $\mathrm{F}_{1} \beta, \mathrm{F}_{1}$-ATPase subunit $\beta ; \mathrm{F}_{0} 9, \mathrm{~F}_{0}$-ATPase subunit 9; GIP, general insertion protein; $\Delta \Psi$, membrane potential. been shown to carry specific targeting information [23-25]. In addition to targeting signals, sorting signals for intramitochondrial protein transport are present in precursor proteins $[23,26]$. Furthermore, non-amino-terminal (carboxyl-terminal) regions of precursors have been shown to contain specific import information [27, 28]. Precursor proteins are unfolded prior to or during translocation into mitochondria $[29,30]$. ATP is needed independently of the requirement for a membrane potential [31]. Recently, the processing peptidase of the mitochondrial matrix and a processing enhancing protein ('PEP'), which is largely associated with the inner membrane, were identified [32]. A major advance in studying the distinct steps of protein import came from the reversible accumulation of precursor proteins at defined stages of their import pathway ('translocation arrest'). This demonstrated the existence of binding sites for precursor proteins on the mitochondrial surface [33-35] and import of precursors via contact sites between both mitochondrial membranes [29]. Sorting of precursors via the matrix space was shown ('conservative sorting') [36].

In this article we characterize distinct steps which have been resolved in protein import into mitochondria. Emphasis is put on the observations obtained by employing translocation intermediates. Our present model on the import pathways of precursor proteins into mitochondria is shown in Fig. 1. The details will be discussed in the following sections.

\section{PRINCIPLES OF GENERATING TRANSLOCATION INTERMEDIATES}

The arrest of a precursor protein at a distinct stage of its translocation pathway across a membrane (or across two membranes as is the case in mitochondria) requires as an essential condition the demonstration that the precursor is on the correct import pathway. This is most convincingly shown 


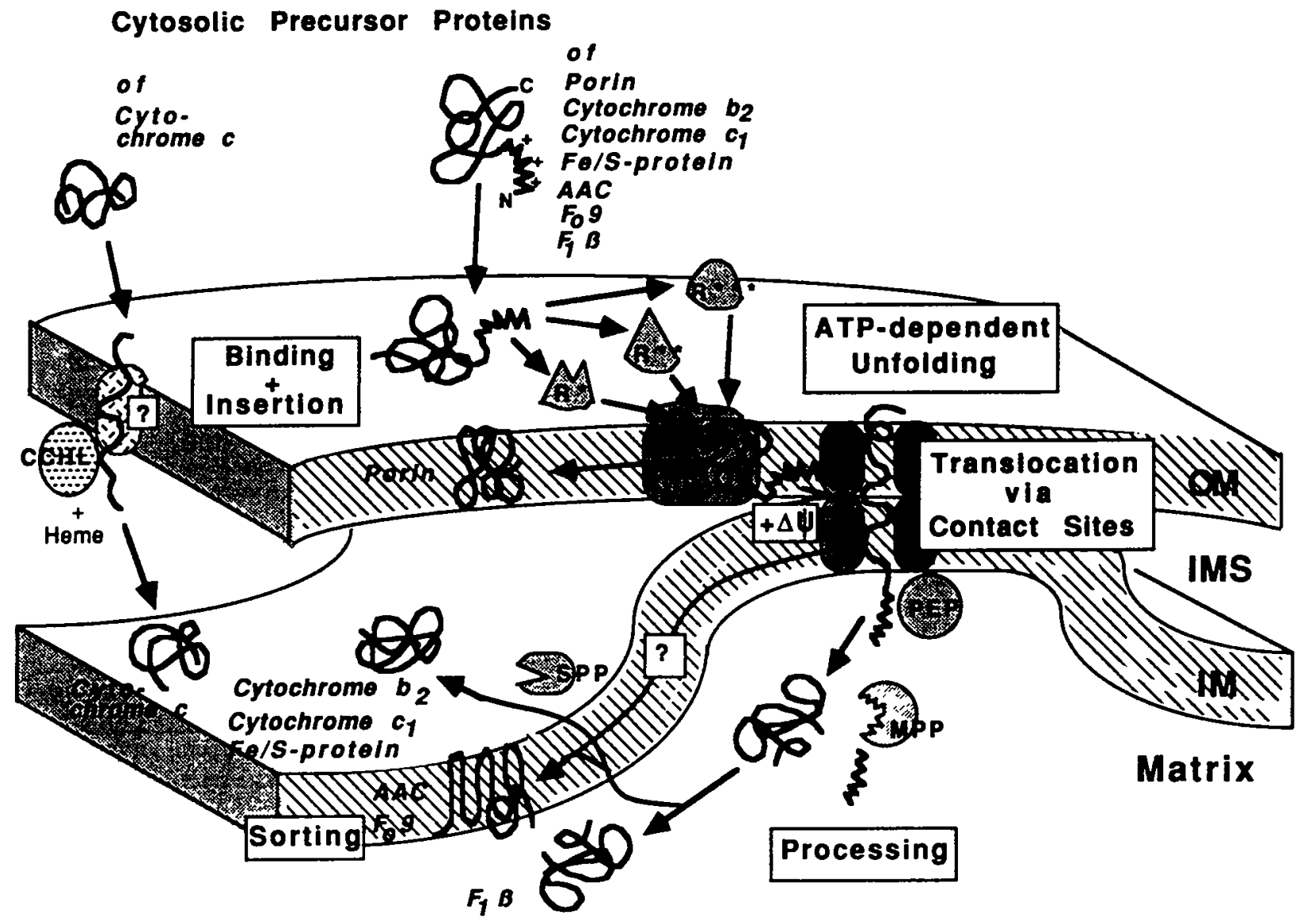

Fig. 1. Working model of the import pathways of precursor proteins into mitochondria. Cytosolic precursor to mitochondrial proteins are specifically recognized by distinct receptor sites $\left(R^{*}, R^{* *}, R^{* * *}\right)$ on the mitochondrial surface. An initial (unspecific) interaction of precursors with lipids of the outer membrane $(\mathrm{OM})$ might precede this step. From the receptor sites, the precursors are transferred to the general insertion protein (GIP) which mediates the initial insertion of precursors into the outer membrane. An ATP-dependent unfolding enzyme, which is located in the cytosol or associated with the mitochondrial membranes, converts the cytosolic precursors into a translocation-competent (unfolded) conformation. The unfolded conformation is required for interaction of the precursors with receptor sites but especially with GIP. Beyond GIP, the import pathways diverge, either to the outer membrane (porin) or to contact sites between outer and inner (IM) mitochondrial membranes. Insertion into the inner membrane requires the membrane potential $(\Delta \Psi)$ across the inner membrane. Two proteins, the matrixprocessing peptidase (MPP) and the processing-enhancing protein (PEP), cooperate to cleave the amino-terminal peptide extensions (targeting signals). Precursors destined for the intermembrane space (IMS) or the inner membrane are then retranslocated across the inner membrane. For some precursors, a second proteolytic processing activity (SPP) is found in the intermembrane space. Prosthetic groups, such as Fe-S clusters or heme, are added to the precursors in the matrix or in the intermembrane space. It can not be excluded so far that some inner membrane proteins move by lateral diffusion from contact sites to their final destination. The import pathway of cytochrome $c$ into mitochondria seems to involve direct translocation of the precursor across the outer membrane. The precursor tightly binds to cytochrome $c$ heme lyase (CCHL) and, after addition of heme, it is released into the intermembrane space. It is not known how cytochrome $c$ is translocated across the outer membrane

by further transport of the precursor on its transport pathway after releasing the translocation arrest. In the case of mitochondria, the precursor has to be completely imported and assembled after release of the arrest. Therefore we have employed the following methods for the reversible arrest of precursors (Fig. 2).

\section{Lowering the temperature}

Import of precursor proteins into mitochondria at low temperatures $\left(0-15^{\circ} \mathrm{C}\right)$ allowed accumulation of precursors at several distinct stages: at the level of binding to receptor sites on the mitochondrial surface $[37,38]$; in contact sites between outer and inner mitochondrial membranes $[29,36$, $37.39,40]$; and in the mitochondrial matrix [41]. The actual stage of accumulation depends on the temperature, the time of incubation, and on additional inhibiting conditions such as dissipation of the mitochondrial membrane potential (for binding of precursors to receptor sites) or inhibition of the processing peptidase in the mitochondrial matrix (for accumulation of precursors in the matrix).

Reducing the levels of nucleoside triphosphates (NTPs) in the import reaction

NTPs (e.g. ATP or GTP) are required for cytosolic unfolding of precursor proteins, probably via an NTP-dependent unfolding enzyme ('translocation competent folding') [42-44]. Precursor proteins which are not sufficiently unfolded are only able partially to insert into the mitochondrial membranes. In the absence of a membrane potential, precursors are accumulated at the initial receptor sites on the mitochondrial surface $[42,45]$; in the presence of $\Delta \Psi$, precur- 


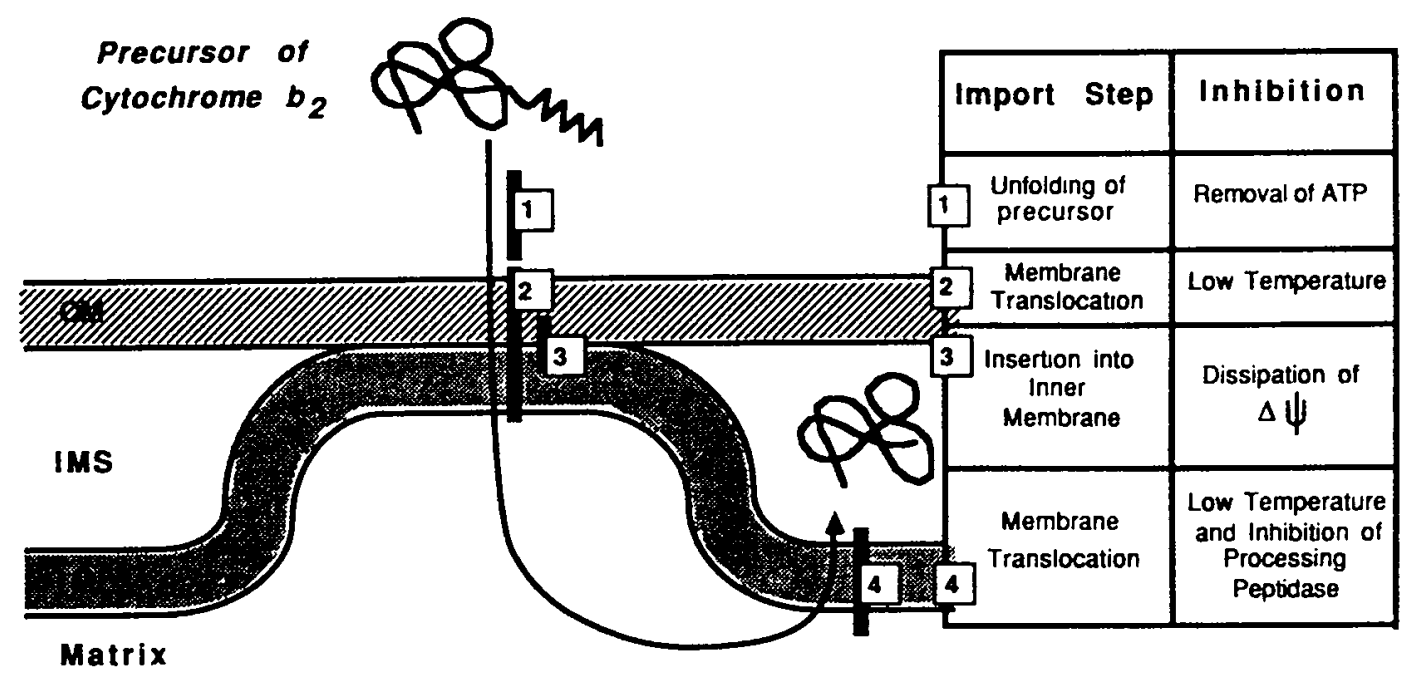

Fig. 2. Methods for arresting a precursor protein at distinct steps of its pathway into mitochondria. The import pathway of the precursor of cytochrome $b_{2}$ from the cytosol via contact sites between mitochondrial outer and inner membranes (OM, IM) into the mitochondrial matrix and then to the intermembrane space (IMS) is shown as an example

sor proteins are trapped in translocation contact sites $[39,42$, 45].

\section{Dissipation of the mitochondrial membrane potential}

The insertion of precursors into the inner mitochondrial membrane requires the presence of the mitochondrial membrane potential. In the absence of the membrane potential, precursor proteins are able to bind to the mitochondrial surface and to insert into the outer membrane $[34,35,37,42,46]$.

\section{Inhibition of the processing peptidase in the mitochondrial matrix}

The metal-dependent processing peptidase in the mitochondrial matrix is inhibited by chelating reagents. Thereby the proteolytic removal of presequences is prevented and precursor proteins can be accumulated in the matrix space [36, 47]. For some precursors, e.g. cytochrome $b_{2}$ and cytochrome $c_{1}$, the translocation arrest in their route to the intermembrane space, or to the outside of the inner membrane, is only partial. To prevent export of a precursor (containing the complete presequence) across the inner membrane, the inhibition of processing is combined with reduction of the temperature [41].

\section{Inhibition of the cytochrome $\mathrm{c}$ heme lyase}

Addition of the heme analogue deuterohemin or omission of NADH inhibits the activity of the cytochrome $c$ heme lyase $[33,48]$. Thereby the precursor of cytochrome $c$ is trapped in a membrane-spanning intermediate position where part of the precursor binds tightly to the cytochrome $c$ heme lyase on the inner side of the outer membrane, while another part of the precursor is still outside the outer membrane $(D$. W. Nicholson and W. Neupert, unpublished results).

After release of the distinct arrested state, the precursor proteins are completely imported and assembled to their functional forms. Lowering the levels of NTPs, dissipation of the membrane potential, or inhibition of cytochrome $c$ heme lyase lead to highly stable translocation arrest. Lowering the temperature, or in several cases inhibition of the processing peptidase, only lead to a retardation of translocation but not to a complete block ('translocation retardation'). In the latter cases, precisely defined reaction conditions are of considerable importance for observation of a distinct translocation intermediate.

\section{Arrest of translocation intermediates with antibodies}

In the cases of receptor-bound or contact-site-embedded intermediates, a stable 'fixing' of the intermediates is also possible by the interaction of the precursors with specific antibodies. When antibodies are pre-bound to carboxyl-terminal domains of precursor proteins, amino-terminal precursor parts are still able to insert into the mitochondrial membranes and are thereby arrested in translocation contact sites [29. 40]. A higher efficiency of intermediate trapping is achieved when the precursors are first accumulated at receptor sites or in contact sites (e.g. at low levels of NTPs) and are then 'tagged' with specific antibodies [45].

\section{UNFOLDING OF MITOCHONDRIAL PRECURSOR PROTEINS}

Precursor proteins can be accumulated in contact sites in a two-membrane-spanning fashion [29]. In this position, the amino-terminal presequences have entered the matrix space where they are proteolytically removed by the processing peptidase in the mitochondrial matrix. Other, probably carboxyl-terminal, portions of the precursors are still outside the outer membrane as demonstrated by their accessibility to proteases added to isolated mitochondria. The distance across the two membranes is larger than the diameter of the assembled and folded polypeptide chain (e.g. $F_{1}$-ATPase subunit $\beta, F_{1} \beta$ ) suggesting that the precursor protein is at least partially unfolded such that it can stretch across both membranes simultaneously. Eilers and Schatz [30] investigated the import of a chimaeric protein between the presequence of cytochrome oxidase subunit IV and the cytosolic protein dihydrofolate reductase. Methotrexate, a specific inhibitor of the latter, was able to bind to the chimaeric protein and thereby prevented import of the protein into mitochondria. A stable tertiary structure, induced by the binding of methotrexate, therefore appears to be incompatible with translocation across the mitochondrial membranes. A similar 
result was obtained when the carboxyl-terminal portion of $F_{1} \beta$ was replaced by copper metallothionein. Binding of copper to the metallothionein moiety (thereby inducing a stable tertiary structure) prevented mitochondrial import of the chimaeric protein [49]. The $F_{1} \beta$-metallothionein chimaera was accumulated at low temperature $\left(4^{\circ} \mathrm{C}\right)$ in contact sites between both mitochondrial membranes (in the absence of copper). Raising the temperature to $30^{\circ} \mathrm{C}$ lead to complete import of the protein. When copper was bound to the protein, while it was trapped in contact sites, further transport into mitochondria was not possible. Thus, folding is also necessary with a precursor protein which is already partially inserted into the mitochondrial membranes.

Depletion of the in vitro mitochondrial import system of NTPs prevents protein import. Re-addition of ATP or of GTP, but not of their non-hydrolyzable analogues, restores import $[31,42,50-52]$. The requirement for NTPs was shown to be independent of the requirement for a membrane potential. A good example of this is the import of porin into the outer membrane, which requires NTPs but not $\Delta \Psi$ [53]. Distinct steps on the import pathway of ADP/ATP carrier and $\mathrm{F}_{1} \beta$ into mitochondria have also been investigated with respect to their dependence on NTPs. Several distinct steps on the import pathways required NTPs as long as part of the precursor was exposed on the surface of the mitochondria. Once the precursor was completely inserted into the outer membrane, further transport into the inner membrane did not need NTPs. Furthermore, two precursors with identical targeting signals but with different carboxyl-terminal protein parts, required different levels of NTPs for import [42]. Similarly, incompletely synthesized polypeptide chains required less NTPs for import than the corresponding completed precursor protein [44]. These results suggested that the essential function of NTPs in mitochondrial protein import is to confer import competence to the precursor proteins, and not an interaction with specific components of the mitochondrial import machinery (e.g. receptor sites).

This hypothesis is supported by the following experiments and results. (a) Precursor proteins synthesized in vitro in rabbit reticulocyte lysates were treated with low concentrations of proteases (in the absence of mitochondria). In the presence of NTPs, the precursors exhibited a higher sensitivity towards proteolytic digest than in the absence of NTPs suggesting that the precursors were in a more loosely folded ('unfolded') conformation [42]. (b) The precursor of porin was subjected to an acid-base treatment followed by rapid neutralization. This converted precursor exhibited a higher sensitivity towards digestion by low concentrations of protease than the authentic precursor, again suggesting a more loosely folded conformation. The mitochondrial import of this converted porin showed the characteristics of the authentic porin precursor, such as binding to receptor sites $[38,54]$, with the striking difference of being independent of NTPs [43].

Recent studies employing mutated precursor proteins suggested somehow contradictory functions of the ATP requirement of mitochondrial protein import. Eilers et al. [55] showed that the fusion protein between a mitochondrial presequence and cytosolic dihydrofolate reductase was unfolded during interaction with the mitochondrial membranes in an ATP-independent manner. The completion of translocation into mitochondria then required ATP. It remains to be elucidated how the unfolding of a cytosolic protein (dihydrofolate reductase) is related to the unfolding of an authentic mitochondrial precursor protein. On the other hand, Chen and Douglas $[56,57]$ constructed mutants of $F_{1} \beta$ which lacked sequences in the mature protein part. The mutants were unable to form the cytosolic tetrameric forms of $F_{1} \beta$ and did not require added ATP for import. Thus, ATP might be involved in dissociation of cytosolic precursor complexes or the mutations might lead to a general conformational alteration which prevents both tetramerization and import-incompetent folding of the precursor. It is therefore not known if these results are a consequence of the special properties of artificial precursor proteins or if they suggest multifunctional roles of ATP for different precursors.

In summary, we conclude that NTPs are required to confer import competence to mitochondrial precursor proteins, probably via conformational alteration ('unfolding') of the precursors. An NTP-dependent unfolding enzyme ('unfoldase'), which may be located in the cytoplasm and/or be associated with mitochondria, was proposed to participate in this process $[42,50,51,58]$. The 'unfoldase' might, for example, be a member of ATP-dependent proteins which accumulate during heat stress of cells ('heat-shock proteins') (for review, see [59]).

\section{SPECIFIC RECOGNITION AND MEMBRANE INSERTION OF PRECURSORS}

Fusion proteins between positively charged amino-terminal sequences of precursor proteins (either the cleavable presequences or mature protein parts of non-cleavable precursors) and non-mitochondrial 'passenger' proteins were shown to be transported into mitochondria. This demonstrated that amino-terminal precursor sequences carry mitochondrial targeting information (for review, see [1]). Recently, we showed that non-amino-terminal (carboxyl-terminal) precursor parts could also carry specific import information. The carboxyl-terminal two thirds of ADP/ATP carrier (a noncleavable precursor) contained sufficient information for import into mitochondria and exhibited all qualitative properties of import of the authentic carrier [27]. Whereas the presequence of $\mathrm{F}_{0}$-ATPase subunit $9\left(\mathrm{~F}_{0} 9\right)$ was sufficient to direct a 'passenger' protein into mitochondria, the presence of the (hydrophobic) mature protein part increased the affinity to binding sites on the mitochondrial surface and accelerated the kinetics of import [28]. We suggest that hydrophobic 'assistant' sequences in mitochondrial precursor proteins act in concert with the positively charged targeting signals to increase the efficiency of import.

Proteinaceous components on the mitochondrial surface are supposed to recognize specifically the targeting signals of precursors and to bind them. The first evidence for this arose from results showing that mild pre-treatment of mitochondria with proteases prevented import of precursors (for review, see [2]). Furthermore, precursor proteins could be accumulated at binding sites in the mitochondrial outer membrane under appropriate conditions. For example, precursors and mitochondria were incubated in the absence of a membrane potential and/or at low temperature. Thereby the precursors of porin, cytochrome $b_{2}$, ADP/ATP carrier and $F_{0} 9$ were accumulated on the mitochondrial surface and could be imported from the binding sites after release of the import block $[28,34,35,38]$. Recently, the binding of ADP/ATP carrier to mitochondria was resolved into two sequential steps $[37,42$, 45]. The precursor first interacts with protease-sensitive receptor sites on the mitochondrial surface where it remains accessible to externally added proteases or antibodies. The precursor then inserts into protease-protected sites in the outer mem- 
brane (named general insertion protein, GIP) where it is protected against externally added proteases or antibodies (Fig. 1). The number of receptor sites is around $0.25 \mathrm{pmol} /$ mg mitochondrial protein, whereas the number of GIP sites is around $2.5 \mathrm{pmol} / \mathrm{mg}$ mitochondrial protein (H.F. Steger, R. Pfaller, N. Pfanner, and W. Neupert, unpublished results). For comparison: porin, the most abundant protein of the outer mitochondrial membrane, is present at about $100 \mathrm{pmol} /$ $\mathrm{mg}$ mitochondrial protein [60]. For the import of porin, similar binding reactions involving two steps were proposed [38]. The number of GIP sites for porin is similar to the number obtained for the GIP sites of ADP/ATP carrier. Interestingly, the precursor of porin competes for the generation of the GIP-associated intermediate of the carrier but not for the generation of the receptor-bound intermediate [38] and (H.F. Steger et al., unpublished results). The concentration of porin required for half-maximal inhibition of generation of the GIPassociated intermediate of ADP/ATP carrier is the same as that required for the inhibition of porin import itself. We conclude that the import pathways of porin and the carrier involve distinct protease-accessible receptor sites which are followed by a common membrane insertion site (GIP). Surprisingly, porin competed for the import of precursor proteins destined for all other mitochondrial compartments, including the Fe-S protein of the $b c_{1}$ complex (intermembrane space), $F_{0} 9$ (inner membrane), and $F_{1} \beta$ (matrix). The present data suggest that the competition again occurs for the interaction with GIP (R. Pfaller, J. Rassow, N. Pfanner, and W. Neupert, unpublished results). We therefore named this site the 'general insertion protein' (GIP). The protease-accessible receptor sites for import of these precursors, which act before GIP, are all sensitive to trypsin or proteinase $\mathrm{K}$. The receptor site for $\mathrm{F}_{1} \beta$, however, is not sensitive to elastase, in contrast to the receptor sites for the other precursors [61].

In summary, we conclude that at least three distinct proteins on the mitochondrial surface act as receptor sites for precursor proteins (one for porin, one for ADP/ATP carrier and one for $F_{1} \beta$ ). They bind their respective precursors and transfer them to the general insertion protein in the outer membrane (Fig. 1). The import of cytochrome $c$ is not competed for by porin. Cytochrome $c$ appears to use an import pathway not involving GIP.

\section{IMPORT VIA TRANSLOCATION CONTACT SITES}

The accumulation of precursors proteins in contact sites between both mitochondrial membranes [29] experimentally demonstrated for the first time a function of the morphologically described sites of close contact between both mitochondrial membranes [62]. (A role of contact sites in protein import had first been proposed by Butow and colleagues [63].) Three distinct methods yielded translocation intermediates spanning both membranes: import at low temperature, pre-binding of antibodies to carboxyl-terminal precursor portions [29] and import at low levels of NTPs [42]. Precursors were thereby trapped in an intermediate position with the presequence in the mitochondrial matrix and other, probably carboxyl-terminal, portions of the precursors outside the outer membrane. The topology of the intermediates was examined by their accessibility to proteases, the matrixprocessing peptidase and externally added proteases, and to externally added antibodies [45]. Thus, both mitochondrial membranes come close enough together to be spanned by a single polypeptide chain (Fig. 1).
Immunocytochemical studies, labelling the contact site intermediates with protein-A-gold particles via the bound antibodies, demonstrated the identity of the morphologically described and of the biochemically defined contact sites. Contact sites appear to be stable structures which can be enriched after subfractionation of mitochondria by sonication [40]. Contact site intermediates are extractable from the membranes with hydrophilic perturbants, such as urea or at alkaline $\mathrm{pH}$. This suggests that mitochondrial precursor proteins are imported through a hydrophilic membrane environment [39]. We propose that specific proteins in contact sites are involved in constituting the architecture of these sites and participate in protein translocation. These functions are fulfilled in tight cooperation with lipids which are probably engaged in structural and possibly also in functional aspects of contact sites.

The insertion of precursor proteins into translocation contact sites requires the membrane potential. The completion of precursor translocation into the inner membrane or matrix is independent of the membrane potential $[29,37]$. As discussed above, binding and insertion into the outer membrane is also independent of the membrane potential. These results suggest that the initial entrance of precursors into the inner membrane, which usually involves the positively charged presequence, is the membrane-potential-dependent import step. On the other hand, we showed that only the electrical component $\Delta \Psi$ of the total protonmotive force and not the chemical component $\Delta \mathrm{pH}$, is required for protein import [46]. We conclude that the membrane potential across the inner membrane (positive outside) exerts an electrophoretic effect on positively charged regions of the precursor proteins thereby triggering the initial entrance of precursors into the inner membrane.

\section{CONSERVATIVE SORTING OF PRECURSORS}

In the mitochondrial matrix, the amino-terminal presequences are proteolytically removed by the matrix-processing peptidase, a soluble protein of $57 \mathrm{kDa}$. A second protein of $52 \mathrm{kDa}$, the processing-enhancing protein, which is largely associated with the inner membrane, markedly stimulates proteolytic processing [32]; it might, for example, bind the precursor proteins and deliver them in a processing-compatible conformation to the matrix-processing peptidase (Fig. 1). Several precursors are proteolytically processed in two steps. The first cleavage is performed by the matrix processing peptidase. The second cleavage is also performed by this peptidase for some precursors [64] or by processing activities located in the intermembrane space for several other precursors [16-18, 41]. Included among the latter precursors are cytochrome $c_{1}$ (whose final location is on the outside of the inner membrane), cytochrome $b_{2}$ and cytochrome $c$ peroxidase (intermembrane space). These precursors contain an uncharged stretch of about 20 amino acid residues in the second part of their presequences $[19,65-67]$ which is involved in the intramitochondrial sorting of the precursors $[1,26,68,69]$. Originally, these hydrophobic sorting domains were assumed to stop transfer of precursors across the inner membrane by interacting with the hydrophobic core of the inner membrane. As will be discussed below, however, this sorting sequence appears to direct the export of precursors from the matrix across the inner membrane. Proteolytic cleavage is not obligatory for translocation across the mitochondrial membranes, since precursors with complete presequences can be accumulated in the mitochondrial matrix and in the intermembrane 
space, albeit with a decreased efficiency in the latter case $[36,41,47]$.

Recent studies [41] have revealed the following import and sorting pathway for cytochrome $b_{2}$ and cytochrome $c_{1}$ to the intermembrane space or to the outside of the inner membrane (Fig. 1). The precursor proteins are imported through a hydrophilic (possibly proteinaceous) membrane environment via translocation contact sites [39] into the mitochondrial matrix. There the positively charged first half of the presequence is removed by the matrix-processing peptidase. The protein with the (hydrophobic) second part of the presequence is then redirected back across the inner membrane. On the outer surface of the inner membrane the second proteolytic cleavage is performed. Cytochrome $b_{2}$ is then released as a soluble protein into the intermembrane space, whereas cytochrome $c_{1}$ is anchored to the inner membrane via a hydrophobic sequence in the mature protein part. These results agree well with the hypothesis of the endosymbiotic origin of mitochondria. In the procaryotic ancestor, the proteins were synthesized in the cytoplasm (corresponding to the mitochondrial matrix) with a signal sequence similar to the hydrophobic second part of the mitochondrial presequence. When genes of the endosymbiont were transferred to the nucleus of the host after the endosymbiotic event, proteins to be translocated into the endosymbiotic organelle were endowed with a hydrophilic mitochondrial targeting signal. Import via contact sites into the mitochondrial matrix was introduced. Thereby the precursors could be translocated into the matrix and could then follow their ancestral sorting and assembly pathways. We have therefore named this import and sorting principle 'conservative sorting' $[36,41]$.

The first example for conservative sorting that was described was the Rieske Fe-S protein of complex III of the respiratory chain, a protein located on the outer surface of the inner membrane [36]. The precursor of the Fe-S protein is imported via translocation contact sites into the matrix where it is processed in two steps to the mature-sized form. (The presequence does not include a longer hydrophobic stretch.) The Fe-S cluster appears to be added to the protein in the matrix (F.-U. Hartl, J. Rassow, and W. Neupert, unpublished results). The mature Fe-S protein is then retranslocated back across the inner membrane and assembled into complex III. Thus, the principle of conservative sorting via the matrix space is similar for Fe-S protein, cytochrome $b_{2}$ and cytochrome $c_{1}$; the difference being that the sorting signal for export from the matrix appears to be contained in the mature part of the Fe-S protein.

It is not known how proteins which are finally embedded with their major parts in the inner membrane are sorted. Preliminary results suggest that at least some of them are also sorted via the matrix space. It cannot be excluded yet whether others move by lateral diffusion from contact sites to their final destination in the inner membrane (Fig. 1).

For some nuclear-encoded proteins, such as the ADP/ATP carrier and the uncoupling protein of the mitochondrial inner membrane, no procaryotic equivalent seems to exist [70]. These proteins might have been introduced into the organelle after the evolutionary endocytosis event [2]. Interestingly, their precursors do not contain a cleavable presequence $[12$, $71,72]$. It is tempting to speculate that precursors which did not descend from components of the endosymbiont carry targeting signal(s) in their mature protein parts.

A clear exception to the principle of conservative sorting is cytochrome $c$, which is located in the intermembrane space. The precursor form, apocytochrome $c$, is synthesized without a cleavable presequence. Its import does not require the membrane potential and it is not significantly competed for by porin $[11,73]$. Import of cytochrome $c$ seems to involve direct translocation across the outer membrane (Fig. 1), although the procaryotic equivalent to cytochrome $c$ is synthesized with a presequence in the cytoplasm of the procaryote and exported across the plasma membrane. After covalent addition of heme by cytochrome $c$ heme lyase, cytochrome $c$ is released as a soluble protein into the intermembrane space $[33,48,74-$ 77]. The import pathway of cytochrome $c$ seems to be a 'short cut' of the complex import pathways of other intermembrane space proteins.

\section{RELATIONSHIP OF MITOCHONDRIAL PROTEIN IMPORT TO PROTEIN TRANSLOCATION ACROSS OTHER CELLULAR MEMBRANES}

The translocation of precursor proteins into or across a membrane is an essential function of many cellular membranes including those of mitochondria, chloroplasts, peroxisomes, the endoplasmic reticulum and procaryotic membranes (for overview, see [78, 79]). In this section we discuss principles of mitochondrial protein import which appear to be of general importance for the understanding of intracellular protein sorting.

\section{Post-translational protein translocation}

The demonstration that mitochondrial protein uptake could occur post-translationally in vivo and in vitro first proved the mechanistic independence of translation and membrane translocation [4-7]. This view is now accepted for practically all membrane systems $[78-85]$.

\section{Requirement for $A T P$}

A requirement for ATP for protein translocation has been shown for chloroplasts [86-88], the endoplasmic reticulum membrane [89-94], peroxisomes [95], and the plasma membrane of Escherichia coli [96-98]. A role for ATP in the unfolding of precursors was suggested for protein translocation into the endoplasmic reticulum [99]. Export of proteins in $E$. coli was shown to require first the unfolding of the precursor protein [100]. An ATP-dependent unfolding of precursors in the cytosol may thus represent a common principle for facilitating protein translocation across different cellular membranes $[43,58,101]$.

\section{Receptors and contact sites}

A protein of the chloroplast envelope (a putative import receptor), which interacts with a chloroplast signal sequence, was found to be localized at contact zones between both membranes of the envelope [102]. Thus, protein import into chloroplasts appears to occur at sites of close contact between both surrounding membranes as it was demonstrated for mitochondria $[29,40]$.

It should be emphasized that, despite the extensive similarities between mitochondrial and chloroplast protein import, the targeting is specific for either organelle. Plant cells did not show any apparent mistargeting of proteins between mitochondria and chloroplasts [103]. The low-efficient mitochondrial import of a precursor carrying a chloroplast targeting signal [104] appears to follow a bypass import path- 
way. It could be demonstrated that the bypass import of a chloroplast protein into mitochondria did not involve protease-accessible receptor sites on the mitochondrial surface. It seemed to enter the protein import pathway at a later stage with a low efficiency (R. Pfaller et al., unpublished results). This bypass import seems to occur in very minor rates, if at all, in vivo [105].

The membrane of the endoplasmic reticulum was the first instance where protein translocation was suggested to occur through a hydrophilic membrane environment [106, 107]. In mitochondria, protein translocation across even two membranes appears to occur through a hydrophilic environment [39].

\section{Requirement for an electrochemical potential}

The requirement of an energized membrane was demonstrated for protein export in $E$. coli (for review see [78, 108]) similar to protein translocation into the inner mitochondrial membrane. It is not clear so far whether the electrophoretic effect on charged precursor sequences, as proposed for mitochondria [46], is also valid for procaryotes $[78,109,110]$.

\section{Conservative sorting}

The best example of mechanistic relationships in protein translocation between mitochondria and other membrane systems is the 'conservative sorting' of precursors $[36,41]$. The basis is that protein export from the mitochondrial matrix appears to be evolutionarily related to protein export in procaryotes, and to protein transport into thylakoids (the inner membranes of chloroplasts) as well as into the inner membranes of photosynthetic bacteria. The signal sequences for all these transport processes appear to be very similar; typically, hydrophobic sequences having a (small) net positive charge. The assumption is that these membrane systems share a membrane of common ancestor, probably a procaryotic-type plasma membrane. The basic mechanisms of protein translocation and assembly have thus been conserved during the evolution of several distinct membrane systems. A (loose) relationship may also exist to the membrane of the endoplasmic reticulum, especially since signal sequences and the leader/ signal peptidase(s) are functionally exchangeable between the endoplasmic reticulum and $E$. coli [111].

For the translocation of nuclear-coded proteins into mitochondria and chloroplasts, a 'new' system was apparently introduced by the eucaryotic cell. Positively charged hydrophilic targeting signals were put at the amino-terminus of the ancestral precursor. These targeting signals direct translocation of the precursors, via contact sites, into the mitochondrial matrix or the chloroplast stroma, respectively. There the hydrophilic targeting signals are removed and now the ancestral signal sequence could engage the conserved transport, folding and assembly pathways into or across the mitochondrial inner membrane or into the chloroplast thylakoids.

In summary, the investigation of mitochondrial protein import has revealed several new insights which have important implications for protein transport processes across other biological membranes. The common aim of these studies is to characterize the molecular events occurring during protein transport across cellular membranes.

We are grateful to all colleagues who have provided us with information prior to publication. We thank Dr D. W. Nicholson for reviewing the manuscript.

\section{REFERENCES}

1. Hurt, E. C. \& van Loon, A. P. G. M. (1986) Trends Biochem. Sci. $11,204-207$.

2. Pfanner, N.\& Neupert, W. (1987) Curr. Top. Bioenerg. 15, 177 219.

3. Nicholson, D. W. \& Neupert, W. (1988) in Protein transfer and organelle biogenesis (Das, R. C. \& Robbins. P. W.. eds) Academic Press, New York, in the press.

4. Hallermayer, G. \& Neupert, W. (1976) in Genetics and biogenesis of chloroplasts and mitochondria (Bücher, T., Neupert, W.. Sebald, W. \& Werner, S., eds) pp. 807-812. Elsevier/NorthHolland, Amsterdam.

5. Harmey, M. A., Hallermayer, G. \& Neupert, W. (1976) in Genetics and biogenesis of chloroplasts and mitochondria (Bücher. T., Neupert, W., Sebald, W. \& Werner, S., eds) pp. 813-818. Elsevier/North-Holland, Amsterdam.

6. Hallermayer, G., Zimmermann, R. \& Neupert, W. (1977) Eur. J. Biochem. 81, 523-532.

7. Harmey, M. A., Hallermayer, G., Korb. H. \& Neupert, W (1977) Eur. J. Biochem. 81, 533- 544.

8. Maccecchini, M.-L., Rudin, Y., Blobel, G. \& Schatz. G. (1979) Proc. Nall Acad. Sci. USA 76, 343-347.

9. Michel, R., Wachter, E. \& Sebald, W. (1979) FEBS Lett. 101, $373-376$.

10. Böhni, P., Gasser, S., Leaver, C. \& Schatz, G. (1980) in The organization and expression of the mutochondrial genome (Kroon, A. M. \& Saccone. C., eds) pp. 423-433. Elsevier North-Holland, Amsterdam.

11. Zimmermann, R., Paluch, U. \& Neupert. W. (1979) FEBS Lett. $108,141-146$.

12. Zimmermann, R., Paluch, U., Sprinzl, M. \& Neupert, W. (1979) Eur. J. Biochem. 99, 247-252.

13. Schleyer, M., Schmidt, B. \& Neupert, W. (1982) Eur. J. Biochem. $125,109-116$.

14. Gasser, S. M.. Daum, G. \& Schatz, G. (1982) J. Biol. Chem. 257, 13034-13041.

15. Kolanski, D. M.. Conboy, J. G.. Fenton, W. A. \& Rosenberg. L. E. (1982) J. Biol. Chem. 257, 8467-8471.

16. Gasser, S. M., Ohashi, A., Daum, G., Böhni, P. C., Gibson. J., Reid, G. A., Yonetani, T. \& Schatz, G. (1982) Proc. Natl Acad. Sci. USA 79, 267-271.

17. Daum, G., Gasser, S. M. \& Schatz, G. (1982) J. Biol. Chem. 257. $13075-13080$.

18. Teintze, M., Slaughter, M., Weiss, H. \& Neupert, W. (1982) J. Biol. Chem. 257, 10364-10371.

19. Kaput, J., Goltz, S. \& Blobel, G. (1982) J. Biol. Chem. 257, $15054-15058$.

20. Vicbrock, A., Perz, A. \& Scbald, W. (1982) EMBO J. l, 565571

21. Schmidt, B., Hennig, B., Zimmermann, R. \& Neupert, W. (1983) J. Cell Biol. 96, 248-255

22. Lewin, A. S. \& Norman, D. K. (1983) J. Biol. Chem. 258,67506755.

23. Hase, T., Müller, U., Riezman, H. \& Schatz, G. (1984) EMBO J. 3. 3157-3164.

24. Hurt, E. C., Pesold-Hurt, B. \& Schatz, G. (1984) FEBS Lett. $178,306-310$.

25. Horwich, A. L., Kalousek, F., Mellman, I. \& Rosenberg. L. E. (1985) EMBO J. 4, 1129-1135.

26. van Loon, A. P. G. M., Brändli, A. W. \& Schatz, G. (1986) Cell $44,801-812$.

27. Pfanner, N., Hoeben, P., Tropschug. M. \& Neupert, W. (1987) J. Biol. Chem. 262, $14851-14854$.

28. Pfanner, N., Müller, H. K., Harmey, M. A. \& Neupert, W. (1987) EMBO J. 6, 3449-3454.

29. Schleyer, M. \& Neupert, W. (1985) Cell 43, 339-350.

30. Eilers, M. \& Schatz, G. (1986) Nature (Lond.) 322, 228- 232.

31. Pfanner, N. \& Neupert, W. (1986) FEBS Lett. 209, 152-156.

32. Hawlitschek, G., Schneider, H., Schmidt, B., Tropschug. M., Hartl, F.-U. \& Neupert, W. (1988) Cell, in the press.

33. Henning, B. \& Neupert, W. (1981) Eur. J. Biochem. 121, $203-$ 212. 
34. Zwizinski, C., Schleyer, M. \& Neupert, W. (1983) J. Biol. Chem. 258, 4071-4074.

35. Riezman, H., Hay, R., Witte, C., Nelson, N. \& Schatz, G. (1983) $E M B O J .2,1113-1118$.

36. Hartl, F.-U., Schmidt, B., Wachter, E., Weiss, H. \& Neupert, W. (1986) Cell 47, 939-951.

37. Pfanner, N. \& Neupert, W. (1987) J. Biol. Chem. 262, 75287536.

38. Pfaller. R. \& Neupert. W. (1987) EMBO J. 6, 2635-2642.

39. Pfanner, N., Hartl, F.-U., Guiard, B. \& Neupert, W. (1987) Eur. J. Biochem. 169, 289-293.

40. Schwaiger, M., Herzog, V. \& Neupert, W. (1987) J. Cell Biol. $105,235-246$.

41. Hartl, F.-U., Ostermann, J., Guiard, B. \& Neupert, W. (1987) Cell 51, 1027-1037.

42. Pfanner, N., Tropschug, M. \& Neupert, W. (1987) Cell 49, 815823.

43. Pfanner, N., Pfaller, R., Kleene, R., Ito, M., Tropschug, M. \& Neupert, W. (1988) J. Biol. Chem. 263, 4049-4051.

44. Verner, K. \& Schatz, G. (1987) EMBO J. 6, 2449-2456.

45. Söllner, T., Pfanner, N. \& Neupert, W. (1988) FEBS Letl. 229, $25-29$.

46. Pfanner, N. \& Neupert, W. (1985) EMBO J. 4, $2819-2825$.

47. Zwizinski, C. \& Neupert, W. (1983) J. Biol. Chem. 258, 1334013346.

48. Nicholson, D. W.. Köhler, H. \& Neupert, W. (1987) Eur. J. Biochem. 164, 147-157.

49. Chen, W.-J. \& Douglas, M. G. (1987) J. Biol. Chem. 262, 15605- 15609 .

50. Chen, W.-J. \& Douglas, M. G. (1987) Cell 49, $651-658$.

51. Eilers, M., Oppliger, W. \& Schatz, G. (1987) EMBO J.6, 10731077.

52. Hartl, F.-U., Ostermann, J., Pfanner, N., Tropschug, M., Guiard, B. \& Neupert, W. (1987) in Cytochrome systems: molecular biology and energetics (Papa, S. et al., eds) pp. 189 196, Plenum Publ. Corp., New York.

53. Kleene, R., Pfanner, N., Pfaller, R., Link, T. A., Sebald, W., Neupert, W. \& Tropschug, M. (1987) EMBO J. 6, 26272633.

54. Pfaller, R., Freitag, H., Harmey, M. A., Benz, R. \& Neupert, W. (1985) J. Biol. Chem. 260,8188-8193.

55. Eilers, M., Hwang, S. \& Schatz, G. (1988) EMBO J. 7, $1139-$ 1145.

56. Chen, W.-J. \& Douglas, M. G. (1987) J. Biol. Chem. 262, 15598-15604.

57. Chen, W.-J. \& Douglas, M. G. (1988) J. Biol. Chem. 263, in the press.

58. Rothman, J. E. \& Kornberg, R. D. (1986) Nature (Lond.) 322, $209-210$.

59. Pelham, H. R. B. (1986) Cell 46, 959-961.

60. Freitag, H., Neupert, W. \& Benz, R. (1982) Eur. J. Biochem. $123,629-636$.

61. Zwizinski, C., Schleyer, M. \& Neupert, W. (1984) J. Biol. Chem. $259,7850-7856$.

62. Hackenbrock, C. R. (1968) Proc. Natl Acad. Sci. USA 61, $589-$ 602.

63. Kellems, R. E., Allison, V. F. \& Butow, R. A. (1975) J. Cell Biol. 65, 1-14.

64. Schmidt, B., Wachter, E., Sebald, W. \& Neupert, W. (1984) Eur. J. Biochem. 144, $581-588$.

65. Sadler, I., Suda, K., Schatz, G., Kaudewitz, F. \& Haid, A. (1984) $E M B O$ J. 3, $2137-2143$.

66. Guiard, B. (1985) EMBO J. 4, 3265-3272.

67. Römisch, J., Tropschug, M., Sebald, W. \& Weiss, H. (1987) Eur. J. Biochem. 164, 111-115.

68. van Loon, A. P. G. M., Brändli, A. W., Pesold-Hurt, B., Blank, D. \& Schatz, G. (1987) EMBO J. 6, 2433-2439.

69. van Loon, A. P. G. M. \& Schatz, G. (1987) EMBO J. 6, 2441 2448.

70. Klingenberg, M. (1985) Ann. N. Y. Acad. Sci. 456, 279-288.

71. Freeman, K. B., Chien, S.-M., Lichtfield, D. \& Patel, H. V. (1983) FEBS Lett. 158, 325-330.

72. Ricquier, D., Thibault, J., Bouillaud, F. \& Kuster, Y. (1983) J. Biol. Chem. 258, 6675-6677.
73. Zimmermann, R., Hennig, B. \& Neupert, W. (1981) Eur. $J$ Biochem. 116, 455-460.

74. Korb, H. \& Neupert, W. (1978) Eur. J. Biochem. 91, 609-620.

75. Hennig, B., Köhler, H. \& Neupert, W. (1983) Proc. Natl Acad. Sci. USA 80, 4963-4967.

76. Veloso, D., Basile, G. \& Taniuchi, H. (1981) J. Biol. Chem. 256, 8646-8651.

77. Taniuchi, H., Basile, G., Taniuchi, M. \& Veloso, D. (1983) J. Biol. Chem. 258, 10963-10966.

78. Wickner, W. T. \& Lodish, H. F. (1985) Science (Wash. DC) $230,400-407$.

79. Zimmermann, R. \& Meyer, D. I. (1986) Trends Biochem. Sci. 11, 512-515.

80. Dobberstein, B., Blobel, G. \& Chua, N.-H. (1977) Proc. Natl Acad. Sci. USA 74, 1082-1085.

81. Wickner, W. (1979) Annu. Rev. Biochem. 48, 23-45.

82. Randall, L. L. (1983) Cell 33, $231-240$.

83. Müller, M. \& Blobel, G. (1984) Proc. Natl Acad. Sci. USA 81, $7421-7425$

84. Lazarow, P. B. \& Fujiki, Y. (1985) Annu. Rev. Cell Biol. 1, $489-$ 530.

85. Just, W. W. \& Hartl, F.-U. (1987) in Peroxisomes in biology' and medicine (Fahimi, H. D. \& Sies, H., eds) pp. 402-416, Springer-Verlag, Berlin.

86. Grossman, A., Bartlett. S. \& Chua, N. H. (1980) Nature (Lond.) $285,625-628$.

87. Flügge, U. I. \& Hinz, G. (1986) Eur. J. Biochem. 160, 563-570.

88. Pain, D. \& Blobel, G. (1987) Proc. Natl Acad. Sci. USA 84, $3288-3292$.

89. Hansen, W., Garcia, P. D. \& Walter, P. (1986) Cell 45, 397 406.

90. Mueckler, M. \& Lodish, H. F. (1986) Nature (Lond.) 322, 549552.

91. Perara, E., Rothman, R. E. \& Lingappa, V. R. (1986) Science (Wash. DC) $232,348-352$.

92. Rothblatt, J. A. \& Meyer, D. I. (1986) EMBO J. 5, $1031-1036$.

93. Waters, M. G. \& Blobel, G. (1986) J. Cell Biol. 102, 1543-1550.

94. Schlenstedt, G. \& Zimmermann, R. (1987) EMBO J. 6, 699703.

95. Imanaka, T., Small, G. M. \& Lazarow, P. B. (1987) J. Cell Biol. 105, 2915-2922.

96. Chen, L. \& Tai, P. C. (1985) Proc. Natl Acad. Sci. USA 82, 4384-4388.

97. Geller, B. L., Movva, N. R. \& Wickner, W. (1986) Proc. Natl Acad. Sci. USA 83, 4219-4222.

98. Yamane, K., Ichihara, S. \& Mizushima, S. (1987) J. Biol. Chem. $262,2358-2362$.

99. Wiech, H., Sagstetter, M., Müller, G. \& Zimmermann, R. (1987) EMBO J. 6, 1011-1016.

100. Randall, L. L. \& Hardy, S. J. S. (1986) Cell 46, $921-928$.

101. Pfanner, N. \& Neupert, W. (1988) in The roots of modern biochemistry (Kleinkauf, H., von Döhren, H. \& Jaenicke, L., eds) pp. 707-712, Walter de Gruyter, Beriin.

102. Pain, D., Kanwar, Y. S. \& Blobel, G. (1988) Nature (Lond.) $331,232-237$.

103. Boutry, M., Nagy, F., Poulsen, C., Aoyagi, K. \& Chua, N.-H. (1987) Nature (Lond.) 328, 340-342.

104. Hurt, E. C., Soltanifar, N., Goldschmidt-Clermont, M., Rochaix, J.-D. \& Schatz, G. (1986) EMBO J. 5, 1343-1350.

105. Pfanner, N., Pfaller, R. \& Neupert, W. (1988) Trends Biochem. Sci. 13, 165-167.

106. Gilmore, R. \& Blobel, G. (1985) Cell 42, 497-505.

107. Evans, E. A., Gilmore, R. \& Blobel, G. (1986) Proc. Natl Acad. Sci. USA 83, $581-585$.

108. Randall, L. L., Hardy, S. J. S. \& Thom, J. R. (1987) Annu. Rey. Microbiol. 4I, 507-541.

109. von Heijne, G. (1986) EMBO J. 5, $3021-3027$.

110. von Heijne, G. (1986) J. Mol. Biol. 192, 287-290.

111. Watts, C., Wickner, W. \& Zimmermann, R. (1983) Proc. Natl Acad. Sci. USA 80, 2809-2813. 